\title{
Clasificación de las propiedades mecánicas de las maderas mexicanas en condición "seca"
}

\author{
Raymundo Dávalos Sotelo ${ }^{1}$ \\ Guadalupe M. Bárcenas Pazos ${ }^{1}$
}

\begin{abstract}
RESUMEN
Como complemento a un sistema de clasificación de las propiedades mecánicas en condición verde desarrollado anteriormente, se creó un sistema de clasificación para las maderas mexicanas libres de defectos en condición seca $(\mathrm{CH}=$ $12 \%$ ), tomando como fuente la misma base de datos. El criterio de clasificación empleado es igualmente la densidad relativa 0 básica. Las propiedades mecánicas consideradas fueron: flexión estática, compresión paralela a la fibra, compresión perpendicular a la fibra, dureza Janka y cortante paralelo a la fibra. Este sistema permite agrupar las maderas en cinco grupos: muy alto, alto, mediano, bajo y muy bajo, mediante una regresión potencial.
\end{abstract}

PALABRAS CLAVE:

Clasificación, maderas, México, densidad relativa, propiedades mecánicas

\section{INTRODUCCION}

Los usuarios de la madera con frecuencia enfrentan la necesidad de comparar entre sí las diferentes maderas existentes en el mercado, con el fin, entre otros, de determinar posibles usos. Se generó previamente un sistema de clasificación o agrupación de las maderas mexicanas en condición verde, tomando como base el conjunto de la información publicada hasta la fecha (Dávalos y

\begin{abstract}
As a complement to a classificaction system created previously for green wood a system was created to classify Mexican woods in clear (defect free) air-dry condition $(\mathrm{MC}=12 \%)$ taking as input the same data base. The classification criterion is, likewise, specific gravity. The mechanical properties considered were: static bending, compression parallel to grain, compression perpendicular to grain, Janka hardness and shear parallel to grain. This system allows to classify woods in five groups: very high, high, medium, low and very low, through a power law regression.
\end{abstract}

KEY WORDS:

Classification, woods, México, specific gravity, mechanical properties

Bárcenas, 1998), en vista de que, en muchos casos, la información publicada únicamente presenta valores de propiedades mecánicas de madera ensayada en condición verde. No existían en la literatura técnica, tablas de clasificación que permitieran hacer este tipo de segregación de las maderas, con contadas excepciones (SARH, 1982; Oxford Forestry Institute -OFI-, 1997), las cuales, además, no abarcan todas las propiedades de interés para los usuarios.

1 Departamento de Productos Forestales y Conservación de Bosques. Instituto de Ecología. Apdo. Postal 63. 91000 Xalapa, Ver. México. barcenas@ecologia.edu.mx.

Manuscrito recibido para su publicación el 7 de junio de 1999. 
En el caso de la madera ensayada en condición seca $(\mathrm{CH}=$ contenido de humedad $=12 \%$ ), en cambio, existen varias opciones publicadas de sistemas de clasificación (véanse por ejemplo, Echenique-Manrique, et al., 1975; OFI, 1997; Fuentes, 1998). Los criterios de clasificación empleados por los diferentes autores varían considerablemente y en términos generales, no son descritos de manera explícita. Pareciera más bien que obedecen a criterios de clasificación arbitrarios.

Tomando como fuente la misma base de datos creada a partir de toda la información publicada acerca de las propiedades tecnológicas de las maderas mexicanas, incluyendo las mecánicas, que se utilizó para la clasificación de madera verde (Bárcenas, Dávalos y Enríquez, 1998), se pueden elaborar tablas de agrupación para madera ensayada en condición seca, empleando los mismos criterios de selección, pero con los valores registrados para madera seca.

\section{OBJETIVOS}

El propósito fundamental de este trabajo es generar un sistema de clasificación o agrupación de las maderas mexicanas en condición seca $(\mathrm{CH}=12 \%)$, tomando como base el conjunto de la información publicada hasta la fecha.

\section{DATOS RECABADOS}

Se acumularon 119 registros de maderas de especies mexicanas (Bárcenas, Dávalos y Enríquez, 1998). Esta información incluye trabajos publicados en revistas periódicas, monografías, tesis profesionales y de posgrado, reportes de investigaciones, memorias de congresos, etc., de la gran mayoría de las especies mexicanas estudiadas hasta 1997. Se tuvo especial cuidado en verificar que no hubiera duplicaciones en los datos capturados y se revisó el conjunto de registros de manera exhaustiva, para evitar los errores de captura.

Las propiedades mecánicas analizadas para la madera libre de defectos fueron: flexión estática (módulo de ruptura y módulo de elasticidad), compresión paralela a la fibra (esfuerzo máximo), compresión perpendicular a la fibra (esfuerzo al límite proporcional), dureza Janka (lateral y extremos) y cortante paralelo a la fibra (esfuerzo máximo).

En aquellos casos en que los valores fueron registrados a contenidos de humedad diferentes al $12 \%$, los valores se ajustaron a dicha cifra, considerando un patrón de variación lineal específico para cada propiedad. Estos patrones de variación, fueron determinados a partir de los porcentajes de variación en la resistencia por cada porciento de contenido de humedad publicados por Markwardt y Wilson (1935) para madera seca de las especies norteamericanas, por la falta de información semejante para maderas nacionales. Es decir, no se tiene una información precisa de cómo varían las propiedades mecánicas de las maderas mexicanas por efecto del contenido de humedad. Aquí se presume que esta variación no difiere sustancialmente de la que presentan maderas de Estados Unidos, aunque no se puede probar esta suposición por el momento.

\section{ANALISIS ESTADISTICOS Y TABLAS DE CLASIFICACION}

Para cada propiedad mecánica analizada, ajustada a un $\mathrm{CH}=12 \%$, se obtuvieron los valores de los parámetros estadísticos usuales: mínimo máximo, promedio, desviación estándar y coeficiente de variación, suponiendo una distribución normal. En la Tabla 1 se incluyen los parámetros correspondientes a las diferentes propiedades. 
Considerando la experiencia desarrollada en un trabajo previo (Dávalos y Bárcenas, 1998), en esta ocasión únicamente se hicieron análisis de regresión con el tipo de ajuste de curvas utilizado en el citado trabajo: la ecuación potencial. Este tipo de curvas tiene la virtud de pasar por el origen de coordenadas, así como de poder ajustarse casi a cualquier conjunto de datos de propiedades mecánicas, generalmente con valores más altos del coeficiente de determinación, $\mathrm{R}^{2}$, que los obtenidos con otros tipos de curvas de regresión.

En todos los casos se empleó la densidad básica o relativa ( $\mathrm{pa} / \mathrm{vv}$ ) como variable independiente. La selección del punto de separación entre grupos se hizo de la misma manera que en el trabajo previo, a partir de una propuesta de Dávalos (1994), desarrollada a partir de consideraciones de diseño estructural, la cual permite que en cada categoría se incluya aproximadamente, un número similar de especies, a excepción del grupo inferior, del cual existen pocos datos registrados y son, además, poco aptas para usos estructurales. Los valores límite de densidad entre los diferentes grupos son $0.3,0.45,0.55$ y 0.7 .

En las figuras 1 a 7 se presentan en forma gráfica los valores de las propiedades mecánicas vs. densidad básica (relativa) de las especies mexicanas, incluyendo las curvas de regresión y las ecuaciones correspondientes con el coeficiente de determinación $R^{2}$. En este trabajo se obtuvieron valores del coeficiente de determinación desde 0.50 para el módulo de ruptura hasta 0.92 para la regresión entre la dureza lateral y la densidad básica.

Utilizando estas ecuaciones es posible predecir los valores de las propiedades mecánicas de la madera libre de defectos de especies forestales mexicanas en condición seca $(\mathrm{CH}=12 \%)$. En la Tabla 2 se incluyen los valores de las propiedades mecánicas determinados como límites entre las diferentes categorías.

\section{COMPARACION CON OTROS SISTEMAS DE AGRUPACION}

La tabla de clasificaciones más frecuentemente citada en México es la de Echenique et al, 1975; recientemente, Fuentes (1998), a partir de una recopilación de información publicada acerca de las propiedades mecánicas de las maderas mexicanas, propuso un nuevo sistema de agrupación. Ambos sistemas son para madera libre de defectos con un contenido de humedad de $12 \%$. También en fechas recientes, se ha publicado una base de datos generada con información publicada de especies a nivel mundial, en formato magnético (OFI, 1997), la cual incluye tablas de clasificación para las propiedades tecnológicas, incluyendo las mecánicas.

Tanto las propuestas de Echenique et al. (1975), como la de Fuentes (1998) no son compatibles con la versión desarrollada aquí, por dos conceptos. En primer lugar, en esos trabajos se proponen seis categorías (Fuentes, 1998) o diez categorías (Echenique et al., 1975) de densidad básica (relativa). Con ambos criterios de agrupación las especies mexicanas no tienen una distribución uniforme entre los grupos, a diferencia del planteamiento propuesto aquí, en el cual la selección de los límites de densidad permite que en cada grupo esté representado un número semejante de maderas.

La segunda diferencia significativa entre los criterios propuestos anteriormente y el descrito aquí, está en las propiedades mecánicas analizadas. El trabajo de Fuentes (1998) incluye tablas de clasificación para las propiedades mecánicas de madera seca, en flexión estática (MOR y MOE), compresión (paralela y perpendicular) y dureza Janka lateral. No incluye tablas para resistencia al cortante paralelo a la fibra ni para dureza Janka en los extremos. El artículo de Echenique et al. (1975), incluye más propiedades: aparte de las mencionadas por Fuentes, las de esfuerzo al límite de 
proporcionalidad en flexión, esfuerzo al límite de proporcionalidad en compresión paralela, dureza Janka en las superficies de los extremos y esfuerzo al momento de la ruptura en cortante paralelo a la fibra. El sistema descrito en las dos publicaciones citadas considera cinco categorías igual que en este escrito.

Las tablas planteadas en la base de datos del OFI (1997) son tanto para madera seca como para madera en condición verde. Presenta una tabla para clasificar madera por densidad con diez categorías. Los rangos de variación en las tablas propuestas en este sistema son muy semejantes a los del trabajo de Echenique et al. (1975). Las mismas razones de incompatibilidad citadas para los trabajos de autores mexicanos aplican para este sistema de origen inglés: no se obtiene una distribución uniforme de las especies y las propiedades mecánicas registradas difieren de las estudiadas aquí. Esta referencia incluye tablas de clasificación para las propiedades mecánicas de madera verde y seca, en flexión estática (MOR y MOE), resistencia en compresión paralela y esfuerzo máximo en cortante paralelo.

\section{CONCLUSIONES}

Se elaboró una serie de gráficas y tablas para clasificar las propiedades mecánicas más importantes de las maderas mexicanas en condición seca $(\mathrm{CH}=12 \%)$. Esta forma de agrupación de las especies facilita el uso apropiado de las maderas al permitir una descripción sencilla, apegada a conceptos tradicionales de asignación de las propiedades a uno de cinco grupos. Esta manera de clasificar a las maderas permite hacer comparaciones directas entre especies, que faciliten la toma de decisiones al momento de recomendar usos potenciales.

Esta clasificación presenta a nuestro juicio, una estructura mas coherente que los sistemas publicados previamente, tanto en México como en el extranjero, pues permite una agrupación basada en datos estadísticos de las propiedades mecánicas de especies de maderas de origen nacional, con el concepto de distribución uniforme entre grupos. Tal vez este sistema sea más útil para fines de agrupación basada en el uso estructural de la madera.

Tabla 1. Parámetros estadísticos de las propiedades mecánicas de maderas mexicanas en condición seca $(\mathrm{CH}=12 \%)$

\begin{tabular}{|c|c|c|c|c|c|c|c|}
\hline \multirow{3}{*}{ VALORES } & \multicolumn{2}{|c|}{$\overline{\text { FLEXION }}$} & \multicolumn{2}{|c|}{ COMPRESION } & CORTANTE & \multicolumn{2}{|c|}{ DUREZA } \\
\hline & \multirow{2}{*}{$\begin{array}{l}\text { Módulo de } \\
\text { Ruptura } \\
\left(\mathrm{kg} / \mathrm{cm}^{2}\right)\end{array}$} & \multirow{2}{*}{$\begin{array}{l}\text { Módulo de } \\
\text { Elasticidad } \\
\left(\mathrm{kg} / \mathrm{cm}^{2}\right)\end{array}$} & Paralela & Perpendicular & Paralelo & \multirow{2}{*}{$\begin{array}{l}\text { Lateral } \\
(\mathrm{kg})\end{array}$} & \multirow{2}{*}{$\begin{array}{l}\text { Extremos } \\
\quad(\mathrm{kg})\end{array}$} \\
\hline & & & $\begin{array}{l}\text { Esf. Máx. } \\
\left(\mathrm{kg} / \mathrm{cm}^{2}\right)\end{array}$ & $\begin{array}{l}\text { Esf. Lím.Prop } \\
\left(\mathrm{kg} / \mathrm{cm}^{2}\right)\end{array}$ & $\begin{array}{l}\text { Esf. Máx. } \\
\left(\mathrm{kg} / \mathrm{cm}^{2}\right)\end{array}$ & & \\
\hline Mínimo & 361 & 43 & 244 & 27 & 49 & 213 & 104 \\
\hline Máximo & 2133 & 251 & 886 & 280 & 213 & 1853 & 1835 \\
\hline Promedio & 993 & 122,569 & 528 & 89 & 113 & 553 & 634 \\
\hline $\begin{array}{l}\text { Desviación } \\
\text { estándar }\end{array}$ & 329 & 34,721 & 137 & 52 & 40 & 340 & 360 \\
\hline $\begin{array}{l}\text { Coeficiente } \\
\text { de variación }\end{array}$ & 33 & 28 & 26 & 59 & 35 & 61 & 57 \\
\hline
\end{tabular}


Tabla 2. Clasificación de características mecánicas de maderas mexicanas (libre de defectos) en condición secada al aire $(\mathrm{CH}=12 \%)$

\begin{tabular}{|l|c|c|c|c|c|||}
\hline \multicolumn{7}{|l|}{} & Muy Bajo & Bajo & Medio & Alto & Muy alto \\
\hline FLEXION & $<550$ & $551-800$ & $801-1000$ & $1001-1300$ & $>1300$ \\
\hline Módulo de Ruptura $\left(\mathrm{kg} / \mathrm{cm}^{2}\right)$ & $<75$ & $76-105$ & $106-125$ & $126-150$ & $>150$ \\
\hline $\begin{array}{l}\text { Módulo de Elasticidad }\left(\mathrm{kg} / \mathrm{cm}^{2}\right) \\
\left({ }^{*} 1000\right)\end{array}$ & & & & \\
\hline COMPRESION & $<325$ & $326-450$ & $451-530$ & $531-650$ & $>650$ \\
\hline PARALELA (E. Máx.) $\left(\mathrm{kg} / \mathrm{cm}^{2}\right)$ & $<35$ & $36-65$ & $66-85$ & $86-125$ & $>25$ \\
\hline PERP. (E. Lím. Prop.) $\left(\mathrm{kg} / \mathrm{cm}^{2}\right)$ & $<50$ & $51-90$ & $91-120$ & $121-165$ & $>165$ \\
\hline CORTANTE & $<50$ & & & \\
\hline Esf. Máx. (kg/cm $\left.{ }^{2}\right)$ & $<150$ & $151-350$ & $351-550$ & $551-900$ & $>900$ \\
\hline DUREZA & $<160$ & $161-400$ & $401-625$ & $626-1050$ & $>1050$ \\
\hline Lateral $(\mathrm{kg})$
\end{tabular}

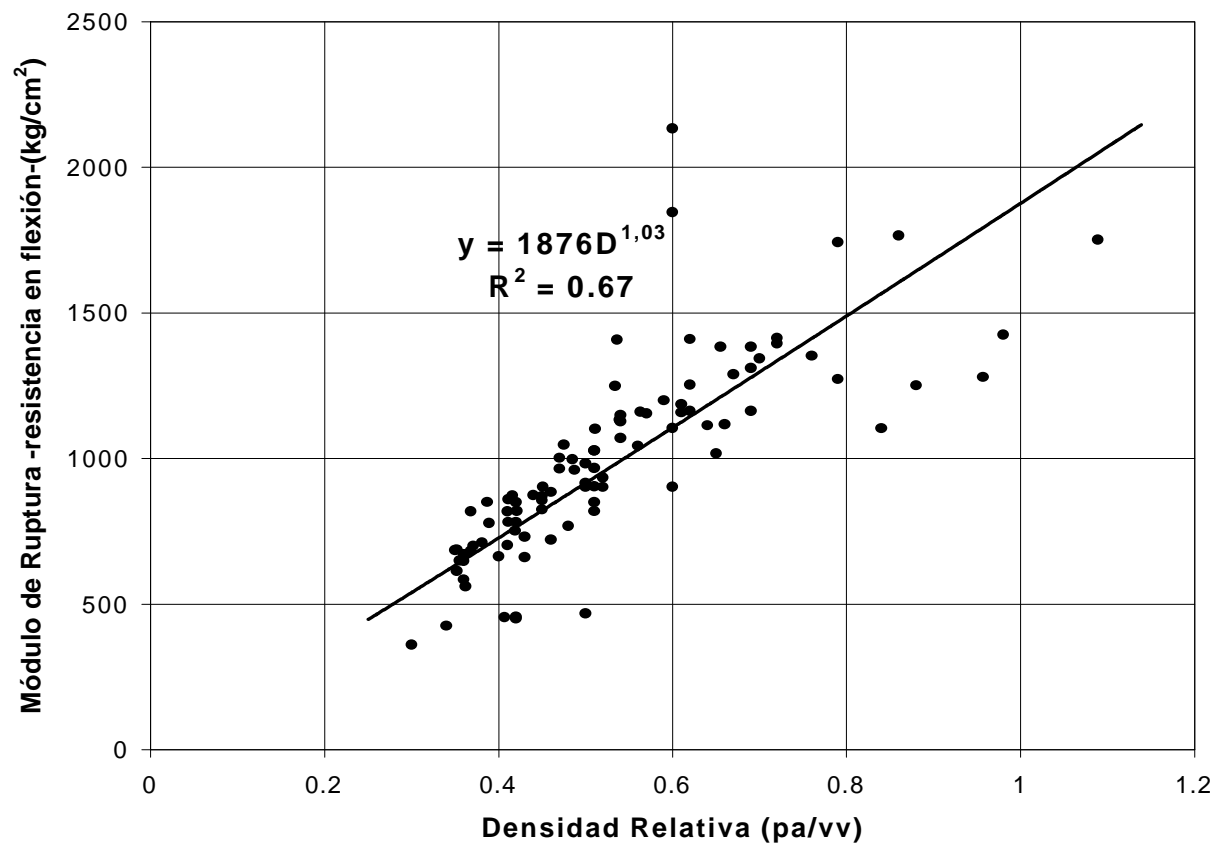

Figura 1. Relación Densidad relativa y Módulo de ruptura para maderas mexicanas en condición seca. 


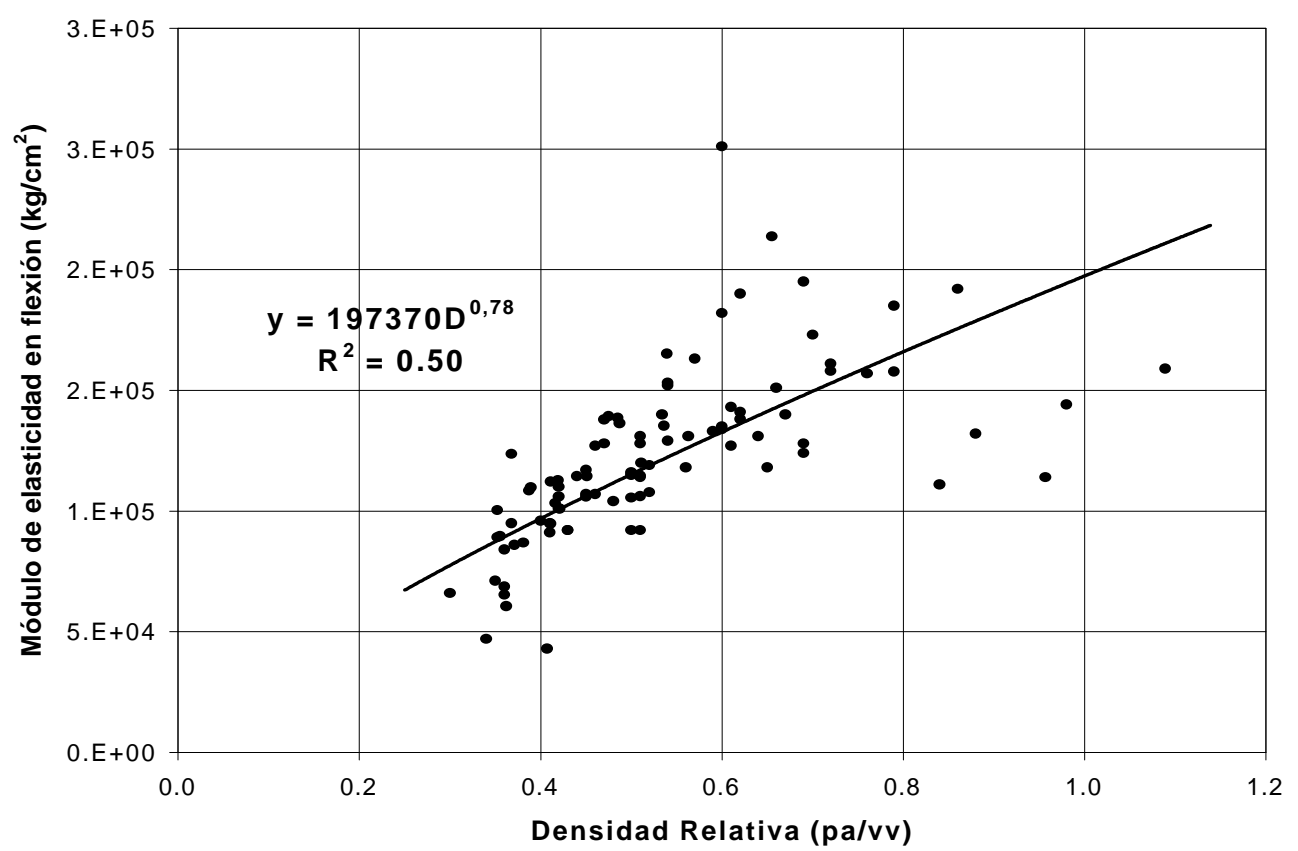

Figura 2. Relación Densidad relativa y Módulo de elasticidad para maderas mexicanas en condición seca.

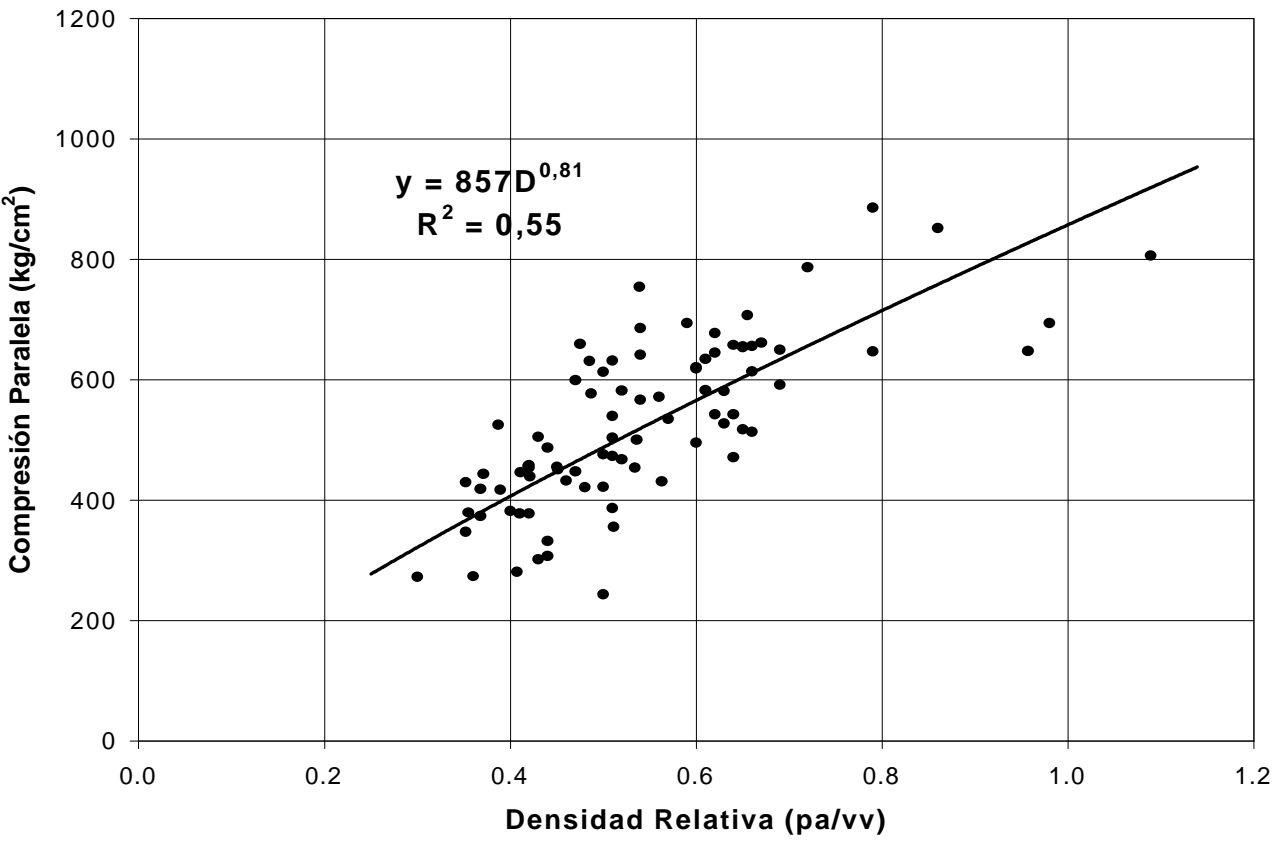

Figura 3. Relación Densidad relativa y Esfuerzo máximo en compresión paralela para maderas mexicanas en condición seca. 


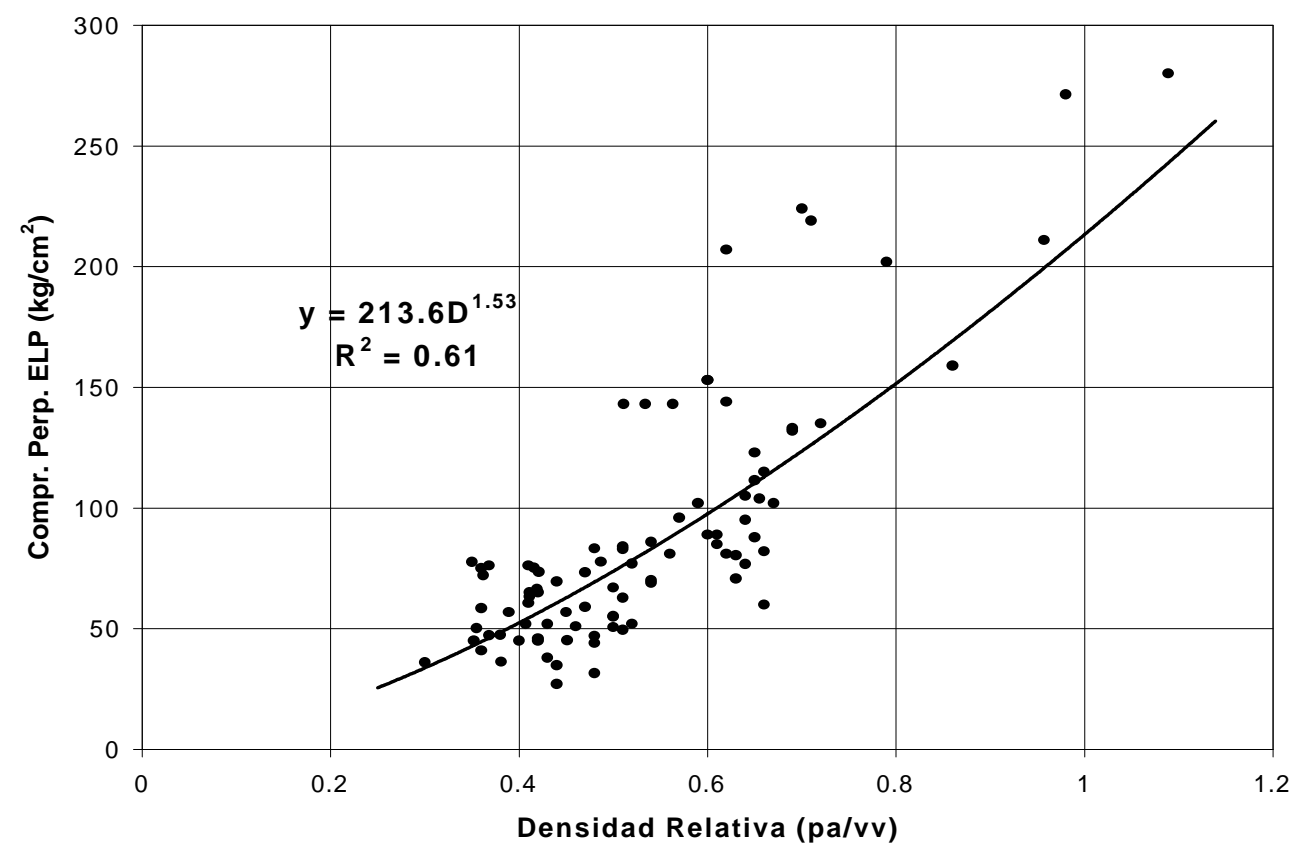

Figura 4. Relación Densidad relativa y Esfuerzo al límite de proporcionalidad en compresión perpendicular para maderas mexicanas en condición seca.

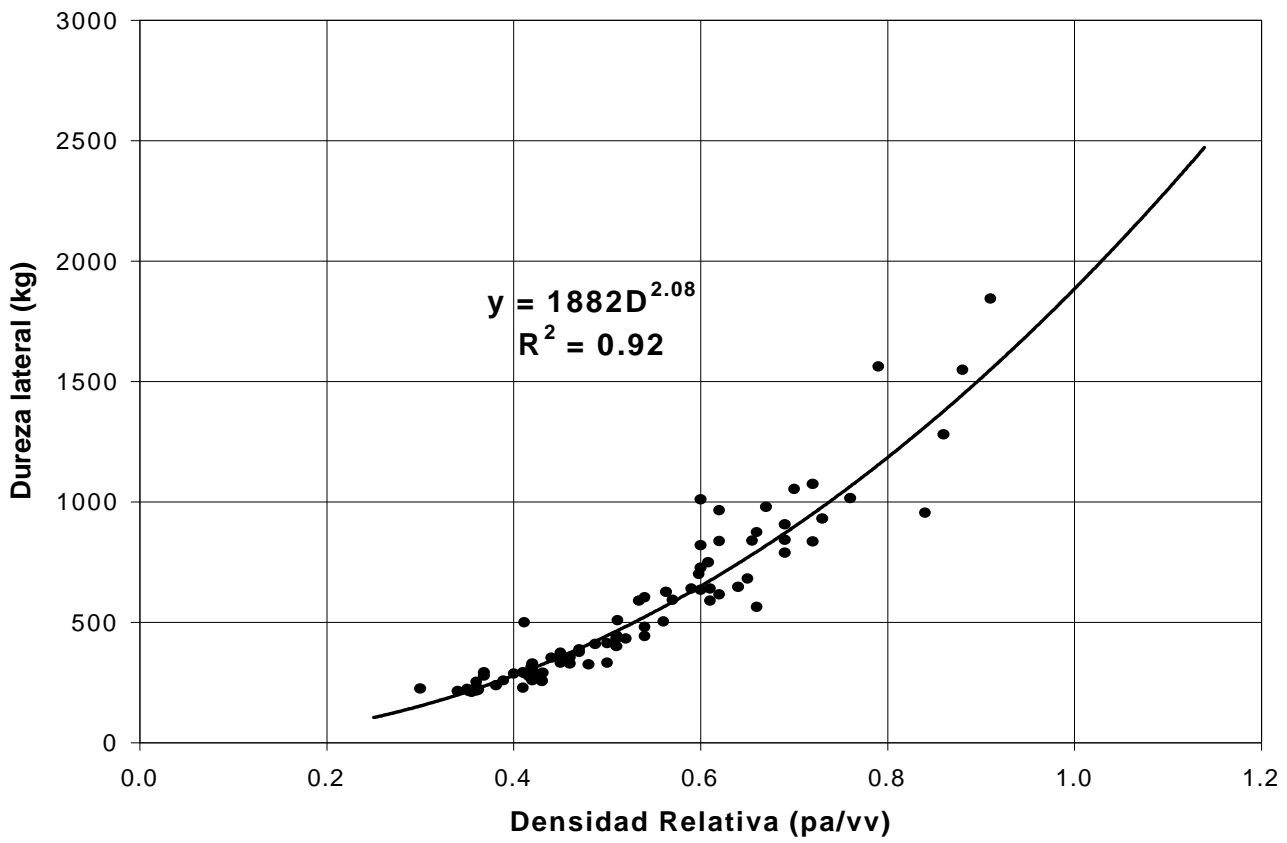

Figura 5. Relación Densidad relativa y dureza lateral para maderas mexicanas en condición seca. 


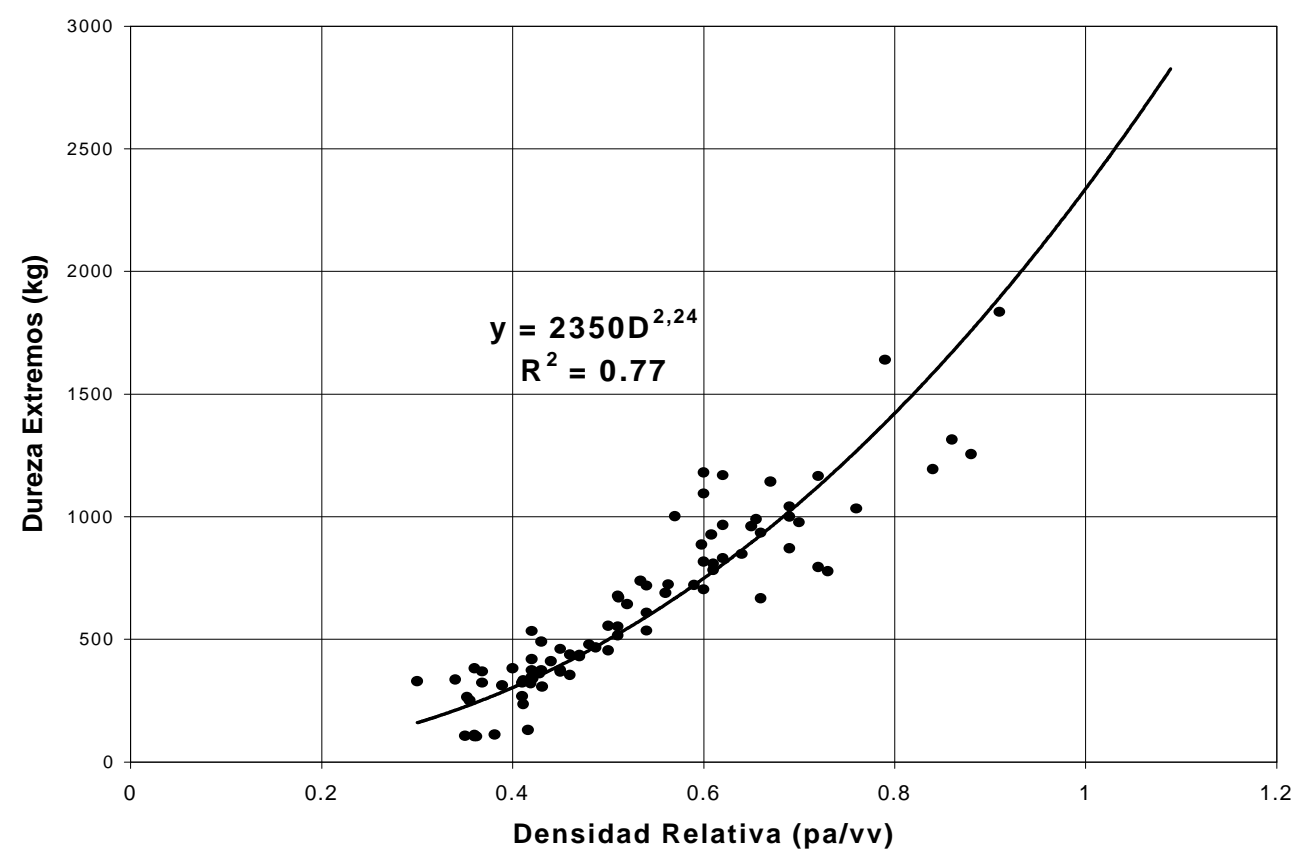

Figura 6. Relación Densidad relativa y dureza extremos para maderas mexicanas en condición seca.

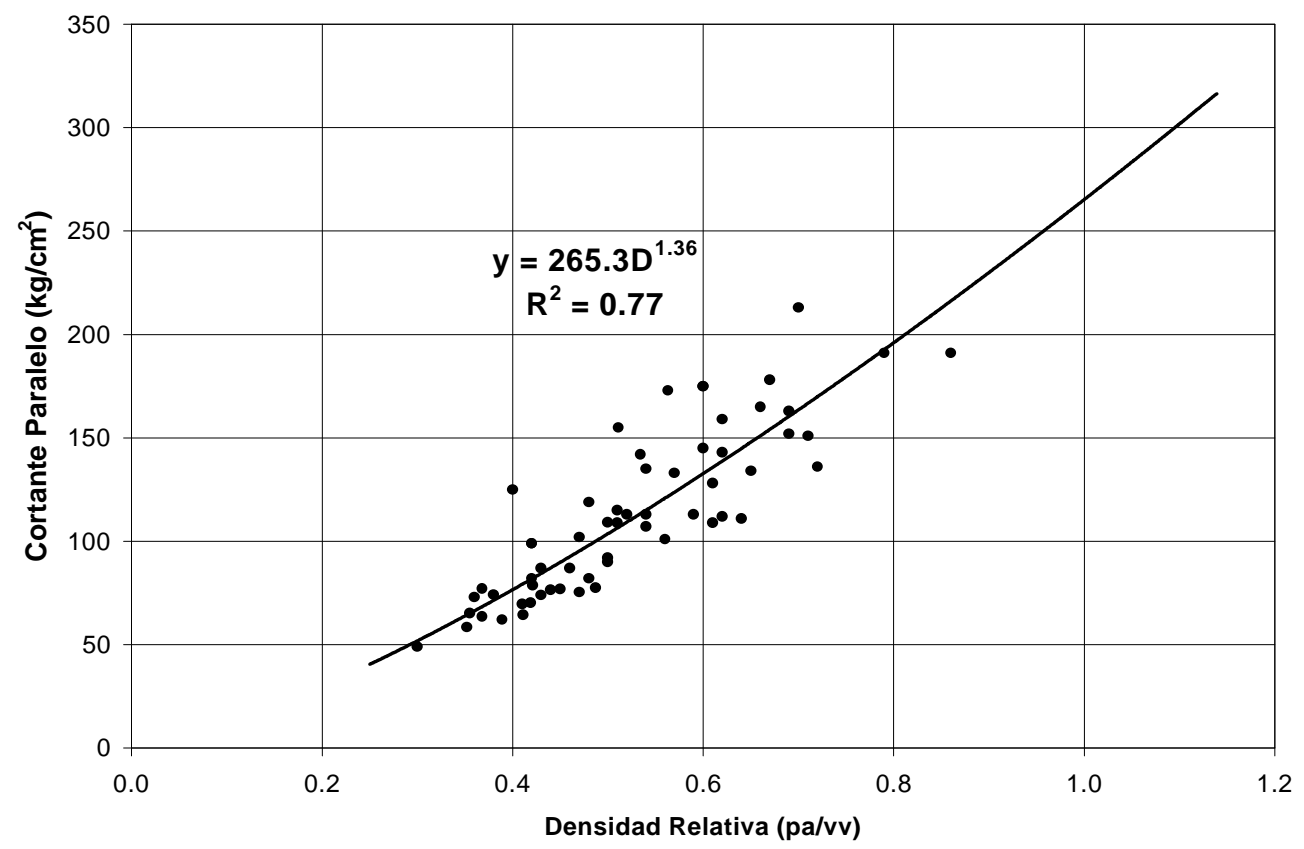

Figura 7. Relación Densidad relativa y Esfuerzo máximo en cortante paralelo para maderas mexicanas en condición seca. 


\section{RECONOCIMIENTOS}

La elaboración de la base de datos que sirvió como fuente de información se concretó con un apoyo del Consejo Nacional de la Biodiversidad (CONABIO): Proyecto clave: K-015 y fondos fiscales concurrentes del Instituto de Ecología, A.C., asignados al Departamento de Productos Forestales y Conservación de Bosques, con el número de clave: 902-13.

\section{REFERENCIAS}

Bárcenas P., G., R. Dávalos S. y M. Enríquez M. 1998. Banco de características tecnológicas de maderas mexicanas. Memorias del Segundo Congreso Mexicano de Tecnología de Productos Forestales. Noviembre 25-27, Morelia, Mich.

Dávalos S., R. 1994. Ayudas de diseño. Cap. 8. Manual de construcción de estructuras ligeras de madera. COFAN. México, D.F.-Ottawa, Ont.Madison, Wis. p:375-418.

Dávalos S., R. y G. Bárcenas P. 1998. Clasificación de las propiedades mecánicas de las maderas mexicanas en condición "verde". Madera y Bosques 4(1):65-70.
Echenique M., R., J. Barajas M., L. Pinzón P. y V. Pérez M. 1975. Características tecnológicas de la madera de diez especies. Estudio Botánico y Ecológico de la Región del río Uxpanapa, Veracruz, No. 1. INIREB. PNIET. Conacyt. México, D.F. p:62.

Fuentes S., M. 1998. Propiedades tecnológicas de las maderas mexicanas de importancia en la construcción. Revista Chapingo Serie Ciencias Forestales y del Ambiente 4(1):221-229.

Markwardt, L.J. y T.R.C. Wilson. 1935. Strength and related properties of woods grown in the United States. USDA Technical Bulletin 479, Washington, D.C.

Oxford Forestry Institute. 1997. Prospect 2.1 for Windows. Department of Plant Sciences. University of Oxford, Inglaterra (Base de datos en archivo magnético: disco compacto).

SARH. 1982. Estudio promocional de 43 especies forestales tropicales mexicanas. Secretaria de Agricultura y Recursos Hidráulicos. SFF, Universidad Edvard Kardelj, Facultad de Biotecnia, Ljubljana, Yugoslavia. $\mathrm{p}: 8$. 
